\title{
Movement and lexical access: Do noniconic gestures aid in retrieval?
}

\author{
SUSAN RAVIZZA \\ University of California, Berkeley, California
}

\begin{abstract}
The production of meaningful gestures has been claimed to enhance lexical access. However, the possibility that meaningless movements also improve retrieval has been largely ignored despite evidence that all types of movements increase with dysfluency. To examine this issue, we conducted two experiments to determine whether movements in general would improve lexical access in a tip-of-thetongue (TOT) paradigm. TOT states were induced by presenting definitions of rare words that participants were then asked to recall. Participants who were required to tap at their own pace while retrieving words obtained significantly higher resolution rates than those who were immobile. Thus, movement does not have to be semantically related to the lexical item in order to aid in retrieval. However, tapping did not improve lexical access in all retrieval tasks. In a lexical retrieval task that relied more on executive abilities (letter fluency), participants who tapped retrieved fewer words than those who were immobile. The fact that movement enhanced lexical access only when retrieval depended on the automatic spread of activation suggests that facilitation may occur because of the activation of neural areas common to both speech and movement.
\end{abstract}

The close relationship between speech and movement is indicated by a multitude of studies demonstrating that the two share common timing and force mechanisms (Bull \& Connelly, 1985; Chang \& Hammond, 1987; Franz, Zelaznik, \& Smith, 1992; Hadar, Steiner, Grant, \& Rose, 1983; Klapp, 1981; Tuller, Kelso, \& Harris, 1982). It is interesting to note, therefore, that movement tends to increase when speech becomes dysfluent (Butterworth \& Beattie, 1978; Dittmann \& Llewellyn, 1969; Ragsdale \& Sylvia, 1982). Ragsdale and Sylvia, for example, reported that $83 \%$ of vocal hesitations in an interview situation were accompanied by movement of some kind and, similarly, $81 \%$ of all movements were associated with vocal hesitations. Given the association of speech and movement, researchers have investigated whether movement helps to restore fluent speech (Beattie \& Coughlan, 1999; Frick-Horbury \& Guttentag, 1998; Graham \& Heywood, 1975; Rauscher, Krauss, \& Chen, 1996; Rime, Schiaratura, Hupet, \& Ghysselinckx, 1984).

Previous studies have been focused on the capacity of one type of movement-iconic/metaphoric gesture-to assist speech fluency. Iconic/metaphoric gestures are movements that contribute semantic information to an utter-

This research was supported by Grants NS 30256 and NS 17778 from the National Institute of Health. The research formed part of the author's $\mathrm{PhD}$ dissertation. I thank my committee members, Rich Ivry, Art Shimamura, Dan Slobin, and John Ohala, as well as Bill Prinzmetal and Julie Fiez for commenting on drafts of this paper. Thanks to Karen Hayes, Paul Aparicio, and Matt Longo for their assistance in running the experiments. Correspondence concerning this article should be addressed to S. Ravizza, University of Pittsburgh, Department of Psychology, 640 LRDC, 3939 O'Hara St., Pittsburgh, PA 15260 (e-mail: ravizza@pitt.edu). ance. For example, we spread our hands apart to demonstrate the largeness of an object. These gestures are assumed to facilitate lexical access because they can provide a cross-modal prime in lexical search (Krauss \& Hadar, 2000). Pretending to slide beads across a wire may help one recall the word abacus by boosting activation of the item through visual/imagistic connections. As such, lexical access should be facilitated only by movements that can represent features of the lexical item. However, some movements that accompany speech are semantically unrelated to the discourse. These noniconic movements (e.g., shifts of posture, biting of nails, drumming of the fingers) have been taken as symptoms of retrieval difficulty (Beattie \& Coughlan, 1999), although only iconic gestures are thought to facilitate retrieval.

The role of iconic gestures in lexical access has been examined by determining the effects of immobilization on speech fluency (Graham \& Heywood, 1975; Rauscher et al., 1996; Rime et al., 1984). Rauscher et al. found that immobile participants spoke more slowly and were more dysfluent when describing spatial aspects of a cartoon than when they were free to move or were describing nonspatial aspects of the cartoon. However, speech fluency can be affected by a number of cognitive and emotional factors other than the ability to make iconic gestures and are, thus, an indirect test of the benefits of iconic gesture to lexical access.

Another approach to studying the role of gesture in lexical retrieval has been to examine the effects of immobilization when people are in the tip-of-the-tongue (TOT) state. In these studies, participants are presented with definitions of rare words and are asked to name them. A word in the TOT state is posited to have insufficient acti- 
vation to engage its phonological constituents (MacKay $\&$ Burke, 1990). Thus, speakers are able to access semantic information about the lexical item but not to produce the word. In contrast to more general measures of speech fluency, such as the number of pauses or speech rate, this procedure allows a more direct measure of lexical access. Using the TOT paradigm, Frick-Horbury and Guttentag (1998) compared lexical retrieval under conditions in which participants were free to move and under those in which movements were prevented by the requirement that participants hold a rod in both hands while depressing a foot pedal. Speakers who were free to move recalled more words overall, but were no different from immobile participants in terms of the number of TOT resolutions.

Although these studies have shown that immobilization impairs fluency and lexical access, none have demonstrated that lexical access is improved because of the iconicity of the movement. Given that immobilization prevents the speaker from performing both iconic and noniconic gestures, it is possible that the ability to make any type of movement will facilitate retrieval. Interestingly, the role of noniconic movements in lexical retrieval has not been explored, despite reports that all types of movements increase with dysfluency (Butterworth \& Beattie, 1978; Dittmann \& Llewellyn, 1969; Ragsdale \& Sylvia, 1982). Indeed, Beattie and Coughlan (1999) noted that TOT trials were often accompanied by all sorts of movements, and the presence of movement was actually used to determine the occurrence of a TOT state, as opposed to reliance on self-reports.

The goal of this research is to determine whether noniconic movements aid in lexical retrieval. The first two experiments examine whether noniconic movement (i.e., tapping) facilitates lexical access in a TOT paradigm. In the third experiment, the role of movement in a letter fluency task is explored.

\section{EXPERIMENTS 1 AND 2}

Experiments 1 and 2 were designed to test whether noniconic movements facilitate the retrieval of words when participants are actively trying to retrieve lexical items. The movements consisted of tapping with both index fingers in synchrony at a self-selected pace. A control condition required the participants to stay as still as possible while holding down two response keys, one with each index finger.

\section{Method}

Participants. Twenty undergraduates at the University of California at Berkeley participated for course credit in Experiment 1. Half of the participants were assigned to the no-movement condition and the rest were placed in the tapping condition. In Experiment 2, 19 undergraduates were paid to participate in a 2-h session while either tapping at their own pace $(n=9)$ or remaining motionless $(n=$ 10).

Stimuli. Two hundred definitions were obtained from previous studies on the TOT phenomenon (Burke, MacKay, Worthley, \& Wade, 1991; Jones, 1989; Yaniv \& Meyer, 1987). ${ }^{1}$
Procedure. Pilot testing indicated that participants could complete about 70 definitions in a 1-h session (Experiment 1) and 165 definitions in a 2-h session (Experiment 2). Definitions were selected in a random order without replacement from the set of 200 items and presented on a computer screen. The participants were instructed to read each definition and, if they knew the answer, to type it in immediately. If they did not know the answer, they were prompted by the computer to rate whether they were in a TOT state. Before the start of the experiment, a TOT state was described to the participants as a feeling that one "knows" a word but is not able to articulate it at the present time. It was emphasized that TOT was not a feeling that one should know the word given one's familiarity with the subject area or general vocabulary skill, but that one does know the word even though one cannot remember it at this moment. Trials in which the participants did not claim to be in a TOT state were categorized as don't know (DK) trials. After rating whether they were in a TOT state, the participants were asked to rate how well they felt they would recognize the answer on a five-point scale $(1=$ definitely would not recognize; $5=$ definitely would recognize).

Once they rated their feeling of knowing (FOK), the participants in both TOT and DK states were again presented with the definition and given $30 \mathrm{sec}$ to retrieve the word. During this time, the participants were instructed either to keep absolutely still or to tap the index fingers of both hands at their own pace. To reduce foot movements, the participants in both conditions were required to depress two foot pedals, one with each foot. In the no-movement condition, the participants were also required to hold down two response keys with their index fingers. Both tapping and immobilization were monitored on two response boards located on the left and right sides of the computer monitor. The computer would indicate an error by beeping if the participants in the no-movement condition lifted their right hands from the response board or if the participants in the tapping condition did not tap once every $5 \mathrm{sec}$. The participants were instructed to type a response if they succeeded in retrieving an answer during this interval. Response times were recorded along with their answers.

\section{Results and Discussion}

The participants immediately knew the answers to approximately half of the questions and definitions. There were no significant differences in TOT, know, and DK rates between conditions $(p s>.1)$ in either Experiment 1 or Experiment 2. A 2 (condition: no movement vs. tapping) $\times 2$ (state: TOT vs. DK) mixed-factor analysis of variance (ANOVA) confirmed that FOK scores were greater when the participants were in a TOT state [Experiment 1: $F(1,18)=$ $183.62, p<.001$; Experiment 2: $F(1,17)=145.49, p<$ $.001]$ than when they were in a DK state, but, in both experiments, neither the main effect of condition nor the interaction of condition and state was significant $(p \mathrm{~s}>.1)$.

The percentage of words ${ }^{2}$ retrieved by the participants in the TOT and DK states during the $30-$ sec interval was measured in each condition (see Figures $1 \mathrm{~A}$ and 1B). Any answer that was entered before $3 \mathrm{sec}$ had elapsed or three taps were recorded was eliminated from the analysis, given that there was little possibility that such retrievals would be influenced by the experimental manipulation.

A 2 (condition) $\times 2$ (state) mixed-factor ANOVA was used to compare resolution rates for the participants in the tapping condition with those for the participants in the nomovement condition. In a comparison of the tapping and no-movement conditions, a main effect of state indicated that more resolutions occurred for the participants in the 

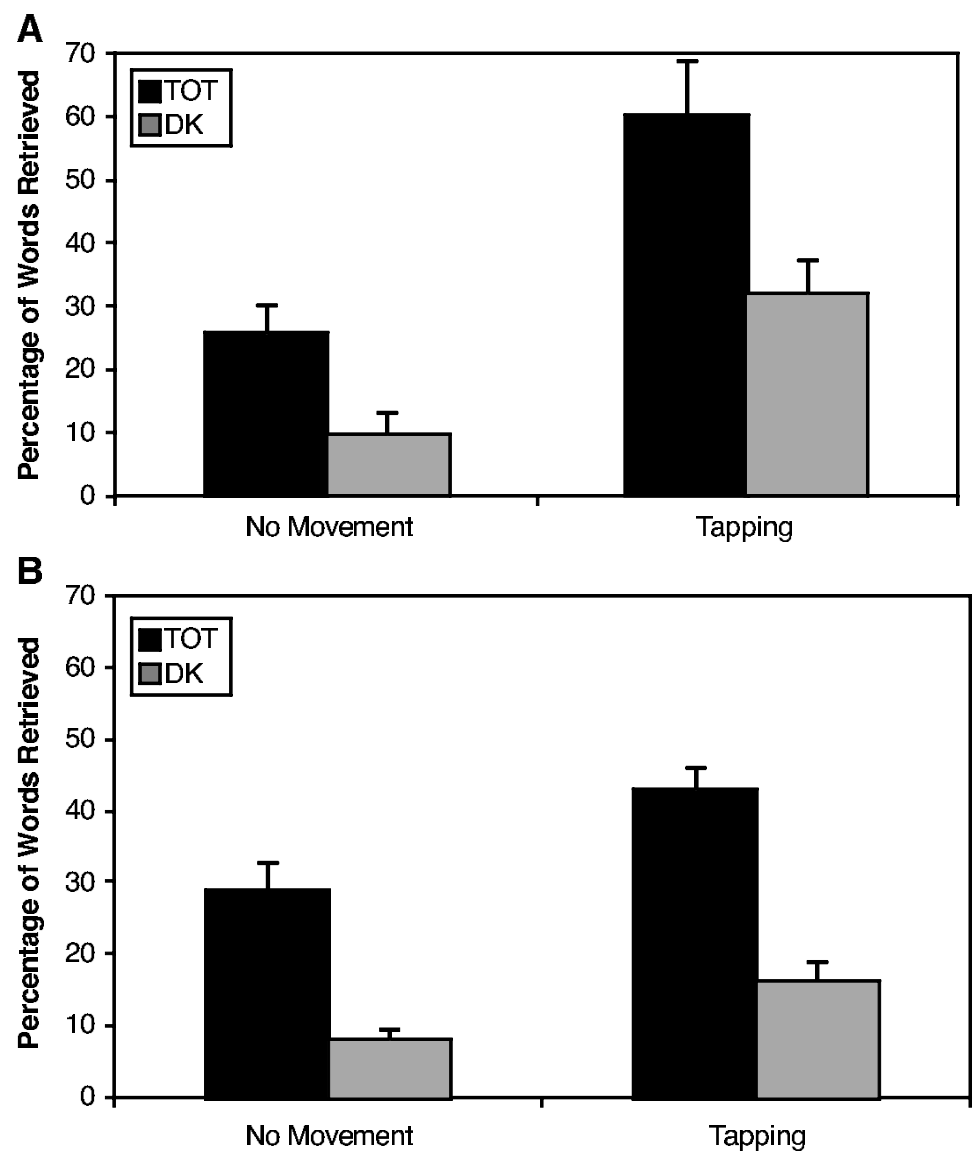

Figure 1. Percentage of words in a TOT or DK state resolved in 30 sec in (A) Experiment 1 and (B) Experiment 2.

TOT state than for those in the DK state [Experiment 1: $F(1,18)=29.57, p<.01$; Experiment $2: F(1,17)=53.54$, $p<.01]$. In both Experiments 1 and 2, significant effects of movement condition were also obtained [Experiment 1: $F(1,18)=16.99, p<.01 ;$ Experiment $2: F(1,17)=17.25$, $p<.01]$ with no interaction effect. The participants in the tapping condition retrieved more words in both states than did those in the no-movement condition. These results support the hypothesis that simple movements do aid in lexical retrieval.

The results of Experiments 1 and 2 demonstrate that, in comparison with the participants in the no-movement condition, those in the tapping condition had improved lexical access. Of course, it is possible that even greater improvements in lexical access would ensue from the performance of iconic gestures. However, it is important to note that movements without semantic content are also effective in increasing resolution rates. Thus, reductions in fluency and lexical access reported in previous research may have been due to the elimination of simple movements rather than to the elimination of iconic gestures.

Interestingly, movement aided retrieval regardless of the subjective state of the participants. Although the par- ticipants were able to retrieve more words while in a TOT state, movement improved resolution rates for words in both the DK and TOT states. The fact that the participants were able to retrieve words that they claimed they did not know underscores the subjective nature of the TOT state. In fact, other studies using the TOT paradigm demonstrate that some proportion of the words in a DK state are correctly recalled (Frick-Horbury \& Guttentag, 1998; Schwartz, Travis, Castro, \& Smith, 2000) or recognized (Schwartz, 2001; Smith, Brown, \& Balfour, 1991). Although words in a TOT state are more likely to be recalled and recognized than words in a DK state, the occurrence of TOT states has been shown to be influenced by variables not related to lexical access, such as the demand characteristics of the experiment (Widner, Smith, \& Graziano, 1996) or the amount of general information known about the target (Schwartz \& Smith, 1997).

The question remains as to why noniconic movements ease lexical access. One explanation may be that movements somehow boost activation levels of lexical items (see Figure 2A). It may be that movement raises the activation level of lexical items that are insufficiently primed when people are in a TOT state. Another explanation for 

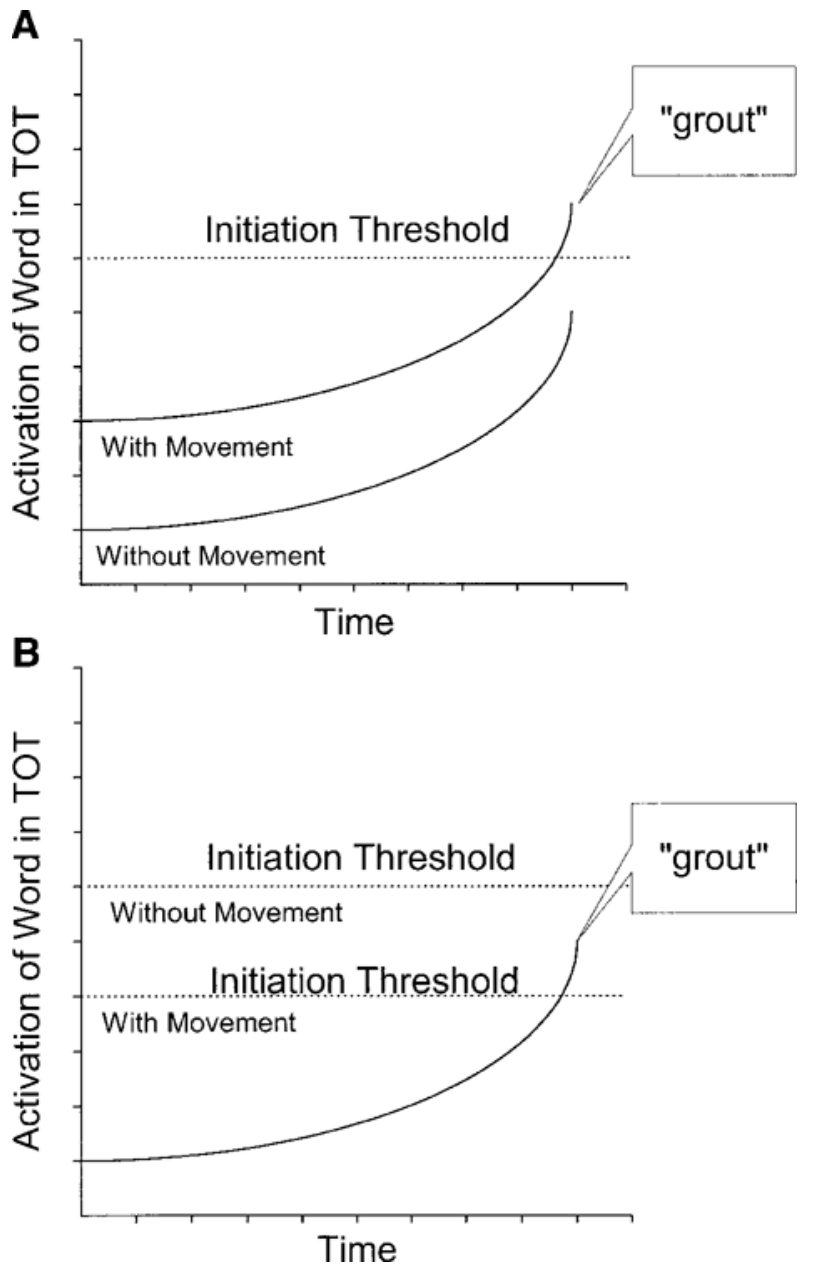

Figure 2. Graphic representation of two explanations that would account for the ability of noniconic movements to aid in lexical retrieval. Panel A demonstrates the possibility that movement boosts activation of a lexical item, whereas Panel $B$ depicts facilitation by the lowering of a generalized response threshold.

the results of Experiments 1 and 2 may be that refraining from movement while retrieving words affects lexical retrieval negatively in comparison with moving. For example, holding still may create a generalized bias not to respond, and so participants are less likely to type in an answer even though they have retrieved it (see Figure 2B). Requiring a participant to move may only lower the threshold for speech initiation and may not be involved in lexical retrieval per se. In order to eliminate any possible response bias, a different paradigm was used in Experiment 3 to assess whether noniconic movements improve lexical access for weakly activated items or simply increase participants' verboseness in all retrieval tasks.

It could also be argued that the participants had a heavier memory load in the no-movement condition because they had to remember to keep still. This argument would be weakened if it were demonstrated that immobile participants are not at a disadvantage in all retrieval tasks. In fact, the results of the next experiment will demonstrate that tapping does not always improve performance.

\section{EXPERIMENT 3}

It is difficult to assess whether noniconic movements actually aid in word retrieval or whether remaining motionless is detrimental to the task. A different retrieval task was used in Experiment 3 to address this issue. Letter fluency tasks require participants to name as many words as they can that start with a specified letter. These tasks are thought to be tests of executive function (Milner, 1964; Moscovitch, 1994), because the ability to generate words relies on the effectiveness of the strategy used to search the lexicon rather than on retrieval per se. Troyer, Moscovitch, and Winocur (1997) found that one type of executive function - the ability to switch retrieval strategieswas highly associated with the number of words generated in a letter fluency task. In contrast, the ability to access the lexicon, as assessed by the degree to which words were phonemically clustered, was less important in letter fluency tasks. Thus, poor performance on letter fluency tasks is thought to originate with deficits in generating or switching search techniques and not because lexical items are weakly activated. If noniconic movements boost weakly activated lexical items, tapping should not improve performance on a letter fluency task. Alternatively, if immobilization causes participants to generally withhold responses or if it places more demands on memory, then people who tap while doing the task should produce more words than those who remain motionless.

\section{Method}

Participants. Thirty-eight undergraduates at the University of California at Berkeley participated in this experiment for course credit. The participants were divided equally between the no-movement and tapping conditions.

Procedure. The participants were given $1 \mathrm{~min}$ to generate aloud as many words as possible that started with the letter Q. Given that the participants were asked to recall rare words in the previous experiments, a less common letter was used in the fluency task to equate retrieval difficulty. The experimenter recorded all answers that were produced. During this initial phase, no instructions were given regarding movement. The participants were then given an extra minute to generate additional words that began with $\mathrm{Q}$ and were told to not repeat words on their original lists. The two-phase fluency task was adopted to make the task demands more similar to those of the TOT experiments. In the preceding experiments, the focus was restricted to trials in which the participants had difficulty in lexical retrieval. In a similar manner, the focus of this experiment was on the performance after the initial flurry of responses.

Preceding the second retrieval interval, the participants were instructed to move or to keep still. The no-movement and tapping conditions were identical to those conditions in Experiments 1 and 2.

\section{Results and Discussion}

The list of words generated by each participant was first examined for errors. Errors included word repetitions and words that had the same root as a previous word with no semantic change (e.g., quake, quaked). The number of unique words generated by each subject was then calcu- 
lated in both retrieval intervals. These scores were analyzed by means of an independent sample $t$ test. There were no significant differences between the groups $[t(24)=0.23, p>.1]$ for the first retrieval interval, but significant differences were obtained in the second interval $[t(24)=2.55, p=.018]$. The participants in the tapping condition retrieved fewer words than did those who were immobile ( 2 vs. 3.85 words).

The results of Experiment 3 stand in sharp contrast to the findings of Experiments 1 and 2. Not only did the tapping group fail to benefit on the fluency task, but they actually performed significantly worse than the immobile participants. These results argue against both alternative hypotheses: that remaining motionless causes participants to inhibit responses and that it is more difficult to remember to hold still than to tap. The benefits of movement are not manifest on all lexical tasks. Rather, they appear to be restricted to situations in which lexical items have already been selected but suffer from insufficient activation.

\section{GENERAL DISCUSSION}

The experiments reported here indicate that "meaningless" movements can actually facilitate lexical retrieval. The participants who tapped at their own pace were able to recall more items in both the TOT and the DK states than were those who remained motionless during retrieval (Experiments 1 and 2). These results demonstrate that noniconic movements facilitate lexical retrieval and that this effect is not due to a general disadvantage of being immobile (Experiment 3). Movement did not result in greater verboseness in general, as would be expected if tapping lowered the threshold of response initiation (see Figure 2B). Noniconic movements do not help in tasks such as letter fluency, in which retrieval depends less on the automatic spread of activation and more on the ability to search the lexicon strategically. Movement may help only in cases in which lexical items have already been selected but suffer from insufficient activation.

One mechanism through which movements facilitate lexical access could be the neural activation of areas used for both language and motor production. For instance, several areas, such as the supplementary motor cortex, basal ganglia, and cerebellum, provide computations that help in producing both speech and movement (Duffy, 1995; Fiez \& Raichle, 1997; Ravizza, 2001). Indeed, a recent imaging study reported activation of the supplementary motor area during TOT retrieval attempts (Kikyo, Ohki, \& Sekihara, 2001). Thus, increased activation to neural areas involved in the planning of both speech and movement may allow bottom-up priming of phonological nodes that are associated with or represented as an articulatory code.

A less interesting explanation of these results could be that the tendency to move differs between the two retrieval tasks, the requirement of moving being more irritating in one task than in the other. For example, if participants have a natural tendency to remain motionless during the letter fluency task, then requiring them to tap may engender a negative emotional state and cause them to retrieve fewer words. In order to rule out this explanation, a new sample of 18 undergraduate and graduate students were videotaped while performing either the TOT task $(n=10)$ described in Experiments 1 and 2 or the letter fluency task $(n=8)$ of Experiment 3 . None of the participants received instructions on how to move, and all knew that they were being videotaped. The numbers of movements made during the first three retrieval intervals of the TOT task (20 sec each) and during the second minute of the letter fluency task were coded. Movements of the head, hands, arms, legs, and feet were summed and found to be equivalent between the two groups $(p>.1)$. In fact, the participants had a tendency to move more during the fluency task than during the TOT task (14 vs. 10.9 movements in $1 \mathrm{~min}$ ). Hand movements were also equivalent between the two types of retrieval tasks (1.75 vs. 2.7). Given that movement rates were comparable in each task, it seems unlikely that differences in the desire to move would have affected lexical retrieval.

Another account for the facilitative role of movement in lexical access could be that it relieves emotional tension associated with the TOT state. In fact, participants in a TOT state report feeling more emotion than those in a DK state (Schwartz, 2001). However, movement aided retrieval to the same degree for participants in both TOT and DK states in Experiments 1 and 2, making this explanation less probable.

Taken together, these experiments have demonstrated that noniconic movements facilitate lexical retrieval for weakly activated items. Although evidence of a facilitative role of noniconic movements in lexical access is provided by these experiments, the hypothesis that this facilitation is due to increased activation in areas shared by speech and movement remains to be tested.

\section{REFERENCES}

Beattie, G., \& Coughlan, J. (1999). An experimental investigation of the role of iconic gestures in lexical access using the tip-of-the-tongue phenomenon. British Journal of Psychology, 90, 35-56.

BRown, R., \& MCNeILl, D. (1966). The "tip of the tongue" phenomenon. Journal of Verbal Learning \& Verbal Behavior, 5, 325-337.

Bull, P., \& Connelly, G. (1985). Body movement and emphasis in speech. Journal of Nonverbal Behavior, 9, 169-187.

Burke, D. M., MacKay, D. G., Worthley, J. S., \& Wade, E. (1991). On the tip of the tongue: What causes word finding failures in young and older adults? Journal of Memory \& Language, 30, 542-579.

Butterworth, B., \& Beattie, G. (1978). Gesture and silence as indicators of planning in speech. In R. N. Campbell \& P. T. Smith (Eds.), Recent advances in the psychology of language 4: Formal and experimental approaches (pp. 347-360). London: Plenum.

Chang, P., \& Hammond, G. R. (1987). Mutual interactions between speech and finger movements. Journal of Motor Behavior, 19, $265-$ 274.

Dittmann, A. T., \& Llewellyn, L. G. (1969). Body movement and speech rhythm in social conversation. Journal of Personality \& Social Psychology, 11, 98-106.

Duffy, J. R. (1995). Motor speech disorders. St. Louis: Mosby.

Fiez, J. A., \& RAichle, M. E. (1997). Linguistic processing. In J. Schmahmann (Ed.), The cerebellum and cognition (pp. 233-254). New York: Academic Press. 
Franz, E. A., Zelaznik, H. N., \& Smith, A. (1992). Evidence of common timing processes in the control of manual, orofacial, and speech movements. Journal of Motor Behavior, 24, 281-287.

Frick-Horbury, D., \& Guttentag, R. E. (1998). The effects of restricting hand gesture production on lexical retrieval and free recall. American Journal of Psychology, 111, 43-62.

Graham, J. A., \& HeYwood, S. (1975). The effects of elimination of hand gestures and of verbal codability on speech performance. European Journal of Psychology, 5, 189-195.

Hadar, U., Steiner, T. J., Grant, E. C., \& Rose, F. C. (1983). Head movement correlates of juncture and stress at sentence level. Language \& Speech, 26, 117-129.

JoNES, G. V. (1989). Back to Woodworth: Role of interlopers in the tipof-the-tongue phenomenon. Memory \& Cognition, 17, 69-76.

Kikyo, H., OHKI, K., \& SeKiHARA, K. (2001). Temporal characterization of memory retrieval processes: An fMRI study of the "tip of the tongue" phenomenon. European Journal of Neuroscience, 14, 887 892.

KLAPP, S. T. (1981). Temporal compatibility in dual motor tasks II: Simultaneous articulation and hand movements. Memory \& Cognition, 9, 398-401.

Krauss, R. M., \& Hadar, U. (2000). The role of speech-related arm/hand gestures in word retrieval. In L. S. Messing \& R. Campbell (Eds.), Gesture, speech, and sign (pp. 93-116). New York: Oxford University Press.

MacKay, D. G., \& Burke, D. M. (1990). Cognition and aging: New learning and the use of old connections. In T. M. Hess (Ed.), Aging and cognition: Knowledge organizationand utilization (pp. 213-263). Amsterdam: North-Holland.

Milner, B. (1964). Some effects of frontal lobectomy in man. In J. Warren \& K. Akert (Eds.), The frontal granular cortex in behavior (pp. 313-334). New York: McGraw-Hill.

Moscovitch, M. (1994). Cognitive resources and dual-task interference effects at retrieval in normal people: The role of the frontal lobes and medial temporal cortex. Neuropsychology, 8, 524-534.

Ragsdale, J. D., \& Silvia, C. F. (1982). Distribution of kinesic hesitation phenomena in spontaneous speech. Language \& Speech, 25, 185 190.

Rauscher, F. H., Krauss, R. M., \& Chen, Y. (1996). Gesture, speech, and lexical access: The role of lexical movements in speech production. Psychological Science, 7, 226-230.

RAVIZZA, S. M. (2001). Relating selective brain damage to impairments with voicing contrasts. Brain \& Language, 77, 95-118.

Rime, B., Schiaratura, L., Hupet, M., \& Ghysselinckx, A. (1984). Effects of relative immobilization on the speaker's nonverbal behavior and on the dialogue imagery level. Motivation \& Emotion, 8, 311-325.
Schwartz, B. L. (2001). The relation of tip-of-the-tonguestates and retrieval time. Memory \& Cognition, 29, 117-126.

Schwartz, B. L., \& SMITH, S. M. (1997). The retrieval of related information influences tip-of-the-tongue states. Journal of Memory \& Language, 36, 68-86.

Schwartz, B. L., Travis, D. M., Castro, A. M., \& Smith, S. M. (2000). The phenomenology of real and illusory tip-of-the-tongue states. Memory \& Cognition, 28, 18-27.

Smith, S. M., Brown, J. M., \& Balfour, S. P. (1991). TOTimals: A controlled experimental method for studying tip-of-the-tongue states. Bulletin of the Psychonomic Society, 29, 445-447.

Troyer, A. K., Moscovitch, M., \& Winocur, G. (1997). Clustering and switching as two components of verbal fluency: Evidence from younger and older healthy adults. Neuropsychology, 11, 138-146.

Tuller, B., Kelso, J. A. S., \& Harris, K. S. (1982). Interarticulator phasing as an index of temporal regularity in speech. Journal of Experimental Psychology: Human Perception \& Performance, 8, 460472.

Widner, R. L., JR., Smith, S. M., \& Graziano, W. G. (1996). The effects of demand characteristics on the reporting of tip-of-the-tongue and feeling-of-knowing states. American Journal of Psychology, 109, 525-538.

YANIV, I., \& MEYER, D. E. (1987). Activation and metacognition of inaccessible stored information: Potential bases for incubation effects in problem solving. Journal of Experimental Psychology: Learning, Memory, \& Cognition, 13, 187-205.

\section{NOTES}

1. Examples of the definitions include "a professional mapmaker" (cartographer), "a bottle designed to be carried in one's pocket" (flask), and "the author of the book 1984" (Orwell).

2 . The measure of a successful lexical retrieval was quite liberal, since it was believed that participants may experience a TOT state for incorrect as well as correct words. For example, one participant retrieved the word observatory although the correct answer was planetarium, and another answered with Laura Ingalls Wilder instead of Louisa May Alcott. Other studies have found that participants can be in a TOT state for an incorrect word anywhere from $21 \%$ to $35 \%$ of the time (Brown \& McNeill, 1966; Burke et al., 1991). Thus, both correct and incorrect answers were scored as successful retrievals.

(Manuscript received June 26, 2001; revision accepted for publication May 8, 2002.) 